\title{
O processo de Indicação Geográfica do Requeijão de Santa Bárbara como entrelaçamento entre a formação técnica e universitária da juventude rural.
}

DOI: 10.37702/2175-957X.COBENGE.2021.3669

Pablo Rodrigo Fica Piras - pafipi@uefs.br

Universidade Estadual de Feira de Santana

Av. Transnordestina $s / \mathrm{n}$

44036-900 - Feira de Santana - BA

ALCIDES DOS SANTOS CALDAS - alcides.caldas@ufba.br

Universidade Federal da Bahia

Av. Cardeal da Silva, n. 303. Edf. Nina Maria. Ap. 902303

40231-305 - Salvador - BA

Géssica Souza de Jesus - gesjsouza@gmail.com

universidade estadual de Feira de Santana

Sitio das Flores 290

44150-000 - Santa Barbara - BA

Rita Ribeiro Lima Menezes - rita_rlm@hotmail.com

Colégio Estadual Professor Carlos Valadares

Avenida Patricio São Paulo 210

44150-000 - Santa Bárbara - BA

MARIA VALDETE VITORIA DA SILVA - valvitoria67@gmail.com

COLEGIO ESTADUAL PROFESSOR CARLOS VALADARES

Av JOSSÉ FALCÃO DA SILVA, COND JOSÉ FALÇÃO BL25

44050-512 - FEIRA DE SANTANA - BA

Maria José de Lima Macedo - zeumacedo@hotmail.com

SEC Bahia

Fazenda Gravatá 50

44150-000 - Santa Bárbara - BA

Ladjane Barbosa dos Santos - ladjane.santos@enova.educacao.ba.gov.br Associação de Pais Professores e Amigos do Colegio Estadual Professor Carlos valadares 
Av Patricio São Paulo, s/n

44150-000 - Santa Barbara - BA

Resumo: Segundo o Fórum de Indicações Geográficas e Marcas Coletivas, o Requeijão de Santa Bárbara é um dos quinze produtos da Bahia que vão na direção da solicitação de registro, concomitantemente com o estímulo à inovação, a competitividade e o desenvolvimento socioeconômico e tecnológico local. Desde o 21 de maio de 2018, com a sessão inaugural do Curso Técnico em Alimentos no Colégio Estadual Professor Carlos Valadares, as iniciativas educativas locais vêm identificando a formação profissional como componente fulcral do desenvolvimento de estratégias de agregação de valor à produção agrícola local e seus derivados. Entre elas, o leite bovino produzido na Bacia do Jacuípe tem o seu destino mais renomado na produção do Requeijão na cidade e ambos se vinculam imageticamente e são tradicionalmente associados uma ao outro. A aproximação das três instâncias que por enquanto participam mais diretamente processo de Indicação Geográfica do Requeijão (além do Colégio Estadual, a Universidade Federal da Bahia e a Universidade Estadual de Feira de Santana) tem possibilitado avançar na estratégia de qualificação técnico-profissional da juventude rural local, em particular com o comprometimento de estudantes dos cursos de Engenharia da UEFS, em aproximações interdisciplinares, na trilha de agregação valor e diferenciação do produto, de aumento do padrão tecnológico e da oferta de emprego e de diversificação da oferta de serviços em setores como o turismo, a gastronomia e o marketing territorial. Apresentam-se antecedentes do percurso até agora, tanto no cenário industrial quanto no educativo local, com informações que pretendem realçar as potencialidades, em uma trajetória complexa e participativa, destinada a preencher os requisitos e atingir o registro, despertando ou confirmando vocações.

Palavras-chave: RDC275; Terroir; Qualificação técnico-profissional; Agregação de valor; Desenvolvimento local. 


\section{O PROCESSO DE INDICAÇÃO GEOGRÁFICA}

DO REQUEIJÃo dE SANTA BÁRBARA COMO ENTRELAÇAMENTO DA FORMAÇÃO TÉCNICA COM A UNIVERSITÁRIA DA JUVENTUDE RURAL.

\section{INTRODUÇÃO}

A criação da Organização Mundial do Comércio (OMC) em 1994, com a participação de 180 nações do planeta, contemplou o Acordo do Direito de Propriedade Intelectual nas atividades comerciais (Acordo TRIPS/ADPIC). Dessa forma, as Indicações Geográficas passaram a ser consideradas uma modalidade de propriedade intelectual, como o são as marcas, patentes, os circuitos integrados de computação, protegidas da concorrência desleal (TRIPS, art. 20), o que abre caminhos para a proteção e valorização de produtos identitários. O Artigo 22 do TRIPS define "indicações geográficas" como indicações que identificam um produto como originário do território, região ou localidade de um Membro, onde uma determinada qualidade, reputação ou outra característica do produto é essencialmente atribuível à sua origem geográfica: a literatura exemplifica com um relógio suíço ou café colombiano (Kennedy, 2005).

No Brasil, segundo a Lei $n^{\circ}$ 9.279, de 14 de maio de 1996 (Brasil, 1996), que regula direitos e obrigações relativos à propriedade industrial no país, em nome do interesse social e do desenvolvimento tecnológico e econômico do País, inicia explicitando que tem como objetivos, entre outros, a "repressão às falsas indicações geográficas" e a "repressão à concorrência desleal". Recentemente, foi divulgado que em 2020 aumentaram em 80 os territórios com potencial para Indicação Geográfica, que se somam aos 77 que já vinham registrados anteriormente. Incluem-se variados produtos: amêndoas de cacau, artesanato, biscoito, cachaça, café, couro, doce, farinha, fruta, queijos, rocha, vinhos, entre vários outros possíveis. Em duas décadas, o número das Indicações Geográficas brasileiras vem crescendo, desde que em 2000 foi registrada a primeira delas, do Vale dos Vinhedos.

As Indicações Geográficas participam de diversas formas nas dinâmicas do desenvolvimento territorial em que estão inseridas. Algumas delas podem ser elencadas a seguir: (1) diferenciam o produto e aumentam o seu valor agregado do produto; (2) estimulam os investimentos na área de produção; (3) valorizam o padrão tecnológico; (4) aumentam a oferta de emprego; (5) estimulam a diversificação dos serviços oferecidos na região; (6) contribuem para a geração de pequenas empresas e novos negócios; (7) estimulam setores como o turismo e a gastronomia; (8) fortalecem o marketing territorial; (9) atenuam ou revertem o êxodo rural; (10) estimulam os investimentos em tecnologia para as variedades produzidas; (11) aumentam a autoestima da população local e o orgulho pelo trabalho exercido; (12) contribuem com valorização da cultura popular, dos saberes locais e das tradições; (13) criam vínculo de confiança com o consumidor, que, sob a etiqueta da IG, sabe que vai encontrar um produto de qualidade; (14) melhoram a comercialização dos produtos, facilitando o acesso aos mercados a partir da propriedade coletiva nacional, até mesmo no mercado internacional; (15) preservam o sistema agroalimentar e as redes sociais associadas; (16) contribuem com a durabilidade econômica, sociocultural e ambiental; (17) dinamizam a economia da região e zonas rurais vinculadas; (18) favorecem a apropriação da dinâmica pelos atores locais, por meio da participação nas deliberações e decisões relativas aos produtos da IG; (19) promovem a preocupação com a garantia da qualidade, da reputação e da identidade do produto; (20) evitam fraudes, uma vez que o registro de IG diferencia um produto dos demais existentes no mercado; (21) contribuem 
para melhorar a renda e qualidade de vida dos produtores associados; (22) favorecem uma repartição igualitária dos benefícios entre produtores associados e a sociedade local; (23) favorecem a Segurança Alimentar - alimento bom, nutritivo, saudável e acessível; (24) estimulam ações para preservação do meio ambiente, com melhoria no uso dos recursos naturais locais, da biodiversidade, da paisagem, dos solos e da água, assumindo compromisso com as gerações futuras; (25) contribuem para a organização dos produtores e suas instituições representativas e de controle.

$\mathrm{Na}$ Bahia, o pioneirismo no incentivo ao processo de discussão e implementação das Indicações Geográficas deve-se à Fundação de Amparo à Pesquisa do Estado da Bahia FAPESB, com o Edital 021/2011, de Apoio a Projetos de Caracterização de Indicação Geográfica (IG) no Estado da Bahia. As cinco propostas apoiadas foram apresentadas por universidades públicas: UESC (Cacau Cabruca - Ilhéus/Itabuna) (Instituto Cabruca); UEFS (Sisal - Valente); UESC (Cerâmicas de Maragogipinho); UESB (Café do Planalto de Conquista); UFBA (Farinha - Nazaré das Farinhas). Dentre elas, até agora, só a do cacau em 2018 obteve o registro de Indicação Geográfica.

O Fórum de Indicações Geográficas e Marcas Coletivas, ao longo de seus encontros, vem organizando os estudos que vêm sendo mediados na direção da solicitação de registros de indicações Geográficas. Além do Requeijão de Santa Bárbara, têm sido identificados outros quatorze: (1) Farinha de Copioba, (2) Azeite da Costa do Dendê, (3) Café de Piatã, (4) Café da Chapada Diamantina, (5) Sisal de Valente, (6) Café do Planalto de Conquista, (7) Banana de Bom Jesus da Lapa, (8) Biscoitos de Vitória da Conquista, (9) Cerâmicas de Maragogipinho, (10) Doces e Geleia de Umbu, (11) Charutos, (12) Pólen de Canavieiras, (13) Rochas Ourolândia, (14) Flores de Maracás).

Os registros de Indicação Geográfica são muito mais frequentes nos produtos alimentícios. Cada levantamento de Identidade Geográfica é um estudo de caso que envolve naturalmente uma triangulação entre paisagem festiva, a ruralidade e o apego ao lugar, no intuito de registrar sensibilidade sobre a natureza do lugar e suas memórias. São, ao mesmo tempo, uma tentativa de superar a introspecção da identidade local no crescimento e preterição do ambiente globalizado, a reinvenção de comunidades que enfrentam transformações sociais e até declínio econômico, e o resgate do Terroir, expressão herdada da vinicultura, que representa a relação simbiótica natureza-cultura, a conexão entre lugares e meios de subsistência, vinculando passado, presente e futuro, em associação com a agricultura, qualidades do solo, clima, práticas e meio local e constitui um ativo importante no desenvolvimento e na construção da identidade do local (Legrand et alii, 2019; Ducros, 2018). A elaboração do processo de registro de Identidade Geográfica trata da valorização e reconstrução de um tecido social comprometido pelos estilos de vida modernos e reaviva uma identidade comum enraizada na ruralidade, sem deixar de perceber que o turismo culinário está em plena expansão (Ducros, 2018). Trata-se, portanto, de um trabalho essencialmente coletivo, multi e interdisciplinar, que envolve uma diversidade de atores e visões complementares.

Esta diversidade de atores nasce precisamente da diversidade de impactos. Vandecandelaere e colaboradores (2018) os analisaram e avaliaram em três grupos: (1) nas empresas: produção, preço e receita; (2) nas cadeias de valor e nos mercados; (3) na dinâmica da agricultura e no desenvolvimento sustentável. Esses autores estabeleceram uma metodologia de acompanhamento e avaliação de nove casos famosos no mundo, mediante descrição do processo e dos resultados e identificação do roteiro que leva aos impactos econômicos, com as principais causalidades dos vários impactos para cada caso, no período definido (Vandecandelaere et alii, 2018).

Deve ser também mencionado que pelo menos dois dos dezessete Objetivos do Desenvolvimento Sustentável - ODSs - da Plataforma Agenda 2030, (2) Fome zero e 
agricultura sustentável, e (12) Consumo e produção responsável foram relacionados pela FAO (2019) quando na valorização das indicações geográficas como um bem coletivo resultado de fatores naturais e/ou humanos na produção de alimentos, pois os agricultores familiares proporcionam por volta de $80 \%$ de todos os alimentos produzidos nos países em desenvolvimento. Assim, tais comunidades desempenham um papel fulcral no fortalecimento da sustentabilidade dos sistemas alimentares locais e globais ao terem 0 poder de preservar e promover seus recursos locais: se lhes for concedido melhor acesso ao mercado para produtos alimentícios de qualidade vinculados ao seu local de origem, essa contribuição será melhor (FAO, 2019).

Santa Bárbara é um município com fontes de renda oriundas principalmente da agricultura e pecuária, com superfície de pouco mais de $347 \mathrm{~km}^{2}$ e aproximadamente 21 mil habitantes, metade deles residentes na zona rural. Com estas características e um PIB de R\$7.770/ano e IDHM de 0,583, grupos de universitários (extensionistas do Programa PIBEX-UEFS e do PET Engenharias UEFS) e de estudantes do ciclo técnico do Colégio Estadual Professor Carlos Valadares, vêm realizando atividades relacionadas com a agregação de valor à produção agrícola local e seus derivados (Souza et alii, 2017; Sena et alii. 2018; Santos et alii, 2020; Dos Santos et alii, 2020a).

Isto posto, no presente trabalho descrevem-se alguns componentes do processo inicial de registro de Indicação Geográfica do Requeijão em Santa Bárbara, sua presença motivadora para a aproximação dos dois níveis de formação (técnica e universitária) da juventude local (rural e urbana), sua incidência como tema transversal já colocado na estratégia de formação profissional em nível Técnico em Agroindústria e Alimentos, a sua convergência com a situação concreta da produção de alimentos e renda local e sua função de elemento de convergência de vários atores participantes no processo de valorização do produto.

\section{METODOLOGIA}

A equipe de estudantes universitários envolvidos no projeto foi integrada por membros do grupo PET Engenharias e de extensionistas do Programa PIBEX-UEFS. Em um dia, separadas em duas equipes, foram visitados os onze produtores locais de requeijão, cada um com diferentes estruturas, distribuídas em onze fabricos em oito localidades (Figura 1). Aplicaram-se dois instrumentos para o levantamento das informações: (1) um questionário estruturado, baseado na RDC275 (Brasil, 2002), com a listagem de verificação de 164 itens dessa norma, junto a um espaço adicional para considerações; (2) um questionário socioeconômico e agroambiental de 51 perguntas, semi-estruturado, auferindo as condições concretas de produção local: produtos, estrutura disponível, tecnologia utilizada, participação no coletivo local de produtores.

Complementarmente, uma equipe de 26 professores do Colégio Estadual Professor Carlos Valadares - CEPCV, desenvolveu um plano de aulas virtuais para todo o Ensino Fundamental e Médio, incluindo os Cursos Técnicos em Agroindústria e Produção Alimentícia, intitulado "Onde moro, onde quero viver: Um olhar econômico, social e ambiental em tempos de pandemia", para ser desenvolvido enquanto a população não tem acesso à vacina contra o vírus SARS-CoV-2. A contextualização da proposta menciona que o projeto visa "buscar por meio da Pesquisa-Ação respostas às demandas das comunidades rural e urbana, de forma a intervir socialmente com o fomento do bem comum, seja este um produto, um processo e/ou um aprimoramento. Propõe-se com isso a integração com o Mundo do Trabalho, o desenvolvimento socioeconômico e ambiental da dinâmica local e territorial, promovendo a intervenção social dos estudantes de diferentes 
séries dentro das comunidades do Município de Santa Bárbara, conforme potencialidades e fragilidades do seu território" (Dos Santos et alii, 2020b). Como parte deste trabalho, foram organizadas as informações da origem e gênero dos jovens barbarenses matriculados no em suas duas formações profissionais (em Agroindústria e em Alimentos) e três modalidades (Regular, Subsequente e PROEJA).

Figura 1 - localização dos fabricos de requeijão em Santa Bárbara

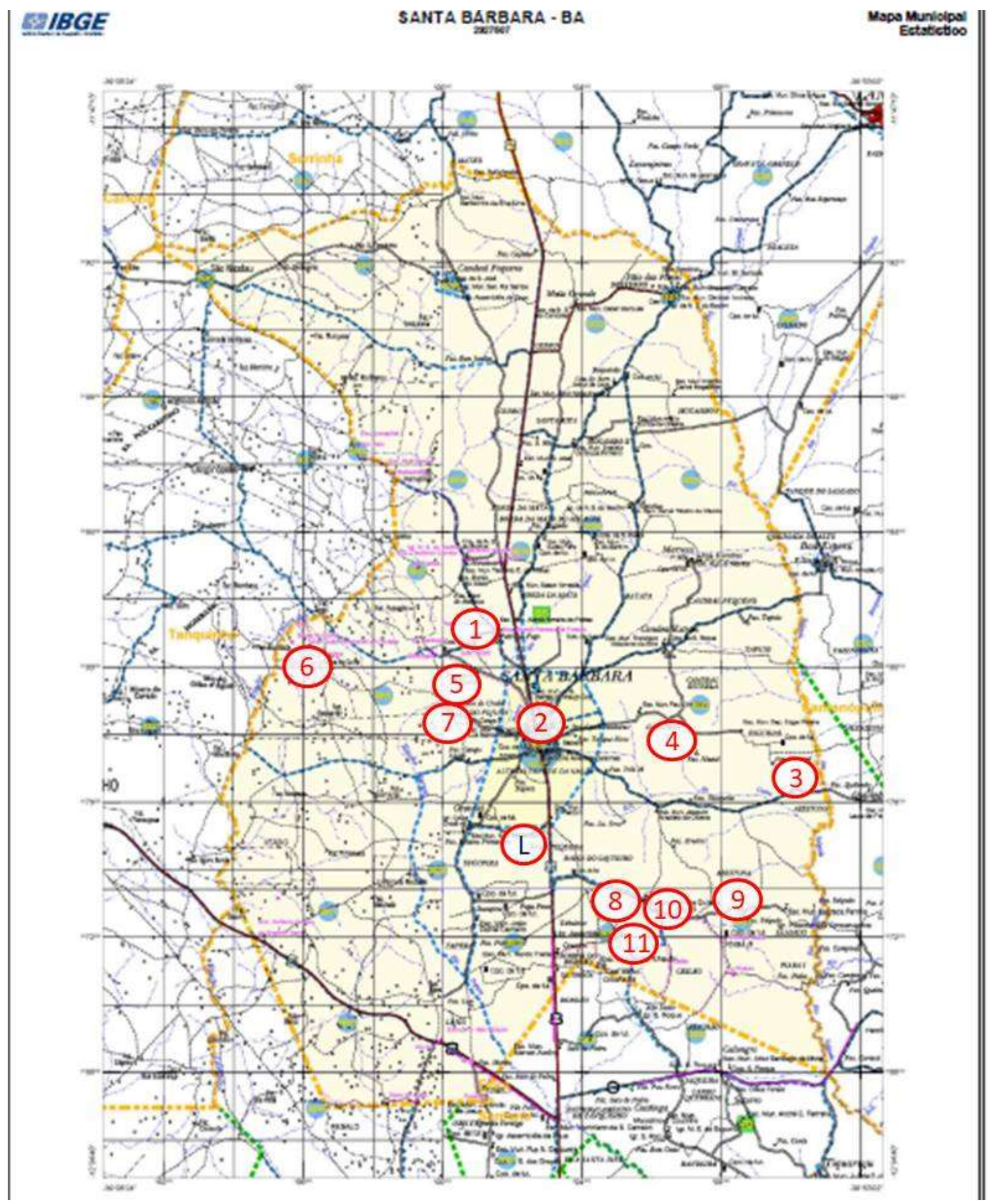

Fonte: Dos Santos et alii (2020a), baseado no mapa municipal de Santa Bárbara (IBGE, s.d.) 


\section{RESULTADOS}

No aspecto do desenvolvimento do trabalho educativo no município de Santa Bárbara, houve liberação de recursos, de acordo com as necessidades previstas para a nova condição didática. O Estado proporcionou 702 chromebooks para os alunos; internet nas escolas estaduais (300Mb); impressões de materiais (módulos específicos); Técnico em informática e cursos para utilização de recursos áudio visuais. O Município proporcionou pontos de acesso à internet nas comunidades rurais, disponibilidade de acesso aos espaços físicos das escolas conforme protocolo, docentes e tablets para os alunos. Ao Colégio coube a organização e divisão em equipes para a execução dos projetos. 0 desenvolvimento dos objetivos traçou-se em quatro linhas de atuação complementares: (1) Produção alimentícia: Educação na produção de alimentos em Santa Bárbara; Boas Práticas são possíveis?; (2) Linguagem: Quem somos, como somos, quantos somos e como surgimos?; (3) Ciências Humanas: Semiárido, contrastes e potencialidades das comunidades de Santa Bárbara/BA; e (4) Exatas: Ciências na comunidade. Ao mesmo tempo que mantém o vínculo entre instituição, docentes e discentes, estas dimensões convergem com os desenvolvimentos necessários para o processo de Indicação Geográfica e torna os estudantes participantes naturais do processo. No conjunto, ao longo do ano, promoveu-se a interação entre a teoria e a prática, a partir da problematização de questões locais, territoriais e estaduais, de forma a aprimorar junto aos estudantes a capacidade de análise, interpretação e resolução dos desafios do mundo, fomentando o empreendedorismo e a intervenção social. Ainda, como elemento de aprofundamento da capilaridade da proposta, foram aumentadas as vagas para o Estágio Civil, no qual os estudantes selecionados são encaminhados para a prática profissional de acordo com 0 projeto adaptado à realidade escolar: colaboração dos alunos por comunidade rural e bairro, com um professor/ funcionário e/ou padrinho do grupo para orientação, aulas e acompanhamento.

Após cadastramento, são 314 os estudantes matriculados nas duas Séries das turmas regulares, nos dois Módulos PROEJA de Técnico em Agroindústria, e nos dois Módulos Subsequentes de Técnico em Alimentos no Colégio Estadual Professor Carlos Valadares - CEPCV (Tabela 1). Deles, dois terços dos estudantes moradores da zona rural do município são do gênero feminino. Em anos anteriores, convergente com a formação de técnico profissional, eles participaram na Feira Escolar de Ciências e Matemática de 2019, na que um eixo mobilizador foi precisamente a Certificação Geográfica do requeijão local. Os títulos dos dez trabalhos apresentados nesta feira já indicam a vocação coletiva para a incorporação tecnológica: (1) "Onde Moro...como quero viver: Qualidade de vida e inclusão"; (2) "Trabalhador Barbarense: braços, sorrisos, corpos e alma"; (3) "Protagonismo Estudantil e a utilização do espaço escolar"; (4) "Saberes populares envolvidos na produção de Requeijão no Município de Santa Bárbara BA"; (5) "Cooperativa de Alimentos Sustentáveis de Santa Bárbara BA (COASSB): Uma proposta empreendedora"; (6) "Desenvolvimento da Cocada de Requeijão: Pacatu, Claricéia e Santa Bárbara; (7) "Idealização e Produção de pizza a Partir da cultura alimentar no Município de Santa Bárbara, BA"; (8) "Um olhar sobre a Feira Livre de Santa Bárbara: Seus segredos, saberes e sabores"; (9) "Elaboração de Requeijão temperado da Cidade de Santa Bárbara"; (10) "Desenvolvimento de novo produto alimentício: Creme de requeijão" (Dos Santos et alii, 2020a). 
Tabela 1: matriculados nos Cursos Técnicos do CEPCV por suas localidades rurais de origem atividade econômica local relevante

(obs: todas as comunidades plantam milho e feijão no período de inverno)

\begin{tabular}{|c|c|c|}
\hline povoado & $\begin{array}{c}\text { matriculados/ } \\
\text { matriculadas/ } \\
\text { total }\end{array}$ & atividades econômicas \\
\hline Água Pequena & $6 / 5 / 11$ & \\
\hline Azeitona & $0 / 0 / 0$ & pecuária leiteira produção \\
\hline Batata & $0 / 2 / 2$ & \\
\hline Boa Vista & $3 / 1 / 4$ & requeijão/apicultura/pecuária leiteira/suinocultura \\
\hline Boqueirão & $2 / 2 / 4$ & hortaliças / agricultura / ovinos \\
\hline Borda da Mata & $0 / 5 / 5$ & \\
\hline Borda da Mata & $2 / 3 / 5$ & \\
\hline Calunga & $0 / 4 / 4$ & \\
\hline Candeal Pequeno & $1 / 1 / 2$ & pecuária / ovinocultura / agricultura familiar \\
\hline Capim Grosso & $1 / 1 / 2$ & \\
\hline Carioca de Baixo & $0 / 2 / 2$ & \\
\hline Cedro & $1 / 0 / 0$ & ovinocultura / agricultura familiar \\
\hline Chapada & $2 / 4 / 6$ & \\
\hline Cruzeiro e Burdão & $0 / 6 / 6$ & avicultura / agricultura familiar \\
\hline Dunda & $0 / 0 / 0$ & hortaliças / agricultura familiar \\
\hline Escorial & $0 / 0 / 0$ & pecuária leiteira \\
\hline Fazenda Campo Limpo & $0 / 2 / 2$ & \\
\hline Fazenda Candeal Estrela & $3 / 4 / 7$ & Pecuária \\
\hline Fazenda Caraíbas & $6 / 6 / 12$ & avicultura / produção de requeijão \\
\hline Fazenda Penha & $2 / 3 / 5$ & \\
\hline Fazenda Rocinha & $0 / 1 / 0$ & agricultura / panificação \\
\hline Fazenda Rogante & $5 / 4 / 9$ & ovinocultura / produção de lanches e salgados \\
\hline Fazenda Varinhas & $0 / 5 / 5$ & suíno e ovinocultura / hortaliças \\
\hline Gravatá & $12 / 20 / 32$ & \\
\hline Lages & $1 / 1 / 2$ & \\
\hline Marreca & $2 / 0 / 2$ & ovinocultura / suinocultura / agricultura familiar \\
\hline Mata Grande & $0 / 2 / 2$ & ovinocultura / agricultura familiar \\
\hline Matão & $1 / 0 / 0$ & hortaliças / pecuária / agricultura familiar \\
\hline Mocambo & $4 / 4 / 8$ & produção de farinha de mandioca / pecuária / ovinocultura \\
\hline Mocó & $2 / 10 / 12$ & \\
\hline Pedra de Fogo & $4 / 8 / 12$ & \\
\hline Pindobeira & $2 / 3 / 5$ & \\
\hline Pojuca & $0 / 3 / 3$ & \\
\hline Saco do Capitão & $2 / 5 / 7$ & \\
\hline Santiago & $1 / 2 / 3$ & \\
\hline São Nicolau & $0 / 0 / 0$ & bar / restaurante / ovinocultura / agricultura familiar \\
\hline Sítio das Flores & $0 / 0 / 0$ & doces / lanches / agricultura familiar / artesanato \\
\hline Sucupira e Papa Pinto & $16 / 23 / 39$ & produção de farinha de mandioca \\
\hline Tabuleiro do Mocó e Campo Limpo & $1 / 2 / 3$ & requeijão / suinocultura / apicultura / batata doce \\
\hline Tapera & $2 / 0 / 2$ & \\
\hline Tiquaruçu & $0 / 3 / 3$ & \\
\hline Tomba & $0 / 0 / 0$ & pecuária leiteira / lanches doces e salgados \\
\hline Varginha & $3 / 1 / 4$ & \\
\hline Vila Feliz & $3 / 2 / 5$ & \\
\hline outros dezenove povoados & $6 / 10 / 19$ & \\
\hline Totais & $96 / 160 / 256$ & \\
\hline
\end{tabular}

Fonte: adaptado e ampliado de Dos Santos et alii (2020a).

No aspecto do levantamento de informações a respeito da produção de requeijão, a equipe de universitários constatou que os produtores atuais estão nucleados em oito localidades, que distam entre 3 e 14 km do centro da cidade, em uma distribuição que se 
concentra na região centro-sul do município. O fornecimento do leite ocorre através de produtores do próprio município e de outros, todos da bacia do rio Jacuípe (foram mencionados Tanquinho, Santanópolis, Capela do Alto Alegre). As quantidades produzidas são muito heterogêneas, com dois produtores, localizados em pontos distantes e próximos às divisas com municípios vizinhos (Santanópolis e Tanquinho) acumulando as maiores quantidades. Todos eles compõem a Cooperativa Mista de Agricultores Familiares e Produtores de Leite de Santa Bárbara - COAFASB, que está recebendo a posse do laticínio construído em Água Pequena, após investimento majoritariamente do governo do Estado. Foi percebido também que a cooperativa não está propendendo a estimular a formação técnica dos seus filiados, para que o uso da estrutura produtiva disponibilizada e enquadrada nos padrões sanitários exigidos pelo órgão estadual seja adequado à pretendido enquadramento nos requisitos legais. A construção do laticínio pressupõe que haverá uso coletivo da estrutura, mas isso não foi confirmado até o momento, aparentemente pela distância dos maiores produtores. Membros do PET Engenharias iniciaram atividades de qualificação em Boas Práticas de Produção e em Identidade Visual de produtos alimentícios, como os processos de Indicação Geográfica requerem.

\section{CONSIDERAÇÕES FINAIS}

Uma estrutura educativa direcionando esforços à percepção das potencialidades e fragilidades do próprio território e promovendo a intervenção social dos seus estudantes naturalmente converge à efetivação de intervenções no campo sociais fomentando o bem comum. Neste contexto, a escolha do Colégio Estadual Professor Carlos Valadares CEPCV de Santa Bárbara do processo de indicação geográfica do requeijão da própria cidade proporciona uma oportunidade ímpar para que um grupo de educação tutorial com prática extensionista participe de ações de entrelaçamento, sobretudo tendo em vista no horizonte a concretização de um objetivo difícil e demorado, mas notoriamente compensador, como é a aquisição do selo supramencionado.

Neste caso da produção de um alimento de amplo reconhecimento popular regional, o grupo PET Engenharias da UEFS, mediante profícua colaboração com os demais atores em tela, se assoma à perspectiva de contribuir a uma narrativa singular, de ocorrência rara, valiosa, interdisciplinar, e que propicia um cenário de aprendizagem significativa para os participantes, estimulando os segmentos estudantis do ensino médio e universitário a envolver-se com o desafio posto. Constatada a necessidade de melhoramento das condições da produção, tanto através de processos educativos como de aprimoramentos na estrutura, e percebidas as numerosas necessidades sequenciais do processo de reconhecimento de Indicação Geográfica, constatamos que esta interação em curso - entre a universidade, uma escola pública com amplo espectro de opções de formação, o poder público municipal e a comunidade local, que direta ou indiretamente participa no sistema de produção em tela - é um espaço privilegiado para a realização do tripé ensino-pesquisaextensão, como definido na Carta Magna, artigo 207, e um exemplo de convergência concreta com o ensino fundamental e médio, como forma de despertar e/ou confirmar vocações e a possibilidade de melhorias no bem-estar local.

\section{REFERÊNCIAS}

Brasil. (1996). Lei no 9.279, de 14 de maio de 1996. Disponível em http://www.planalto.gov.br/ccivil 03/leis/19279.htm. Acesso em 09 de maio de 2021. 
Brasil/ANVISA - Agência Nacional de Vigilância Sanitária, Brasil. (2002). Resolução RDC no 275, de 21 de outubro de 2002. Dispõe sobre o Regulamento Técnico de Procedimentos Operacionais Padronizados aplicados aos Estabelecimentos Produtores/Industrializadores de Alimentos e a Lista de Verificação das Boas Práticas de Fabricação em Estabelecimentos Produtores/Industrializadores de Alimentos. Disponível em http://portal.anvisa.gov.br/wps/wcm/connect/dcf7a900474576fa84cfd43fbc4c6735/R $\mathrm{DC}+\mathrm{N} \% \mathrm{C} 2 \% \mathrm{BA}+275,+\mathrm{DE}+21+\mathrm{DE}+\mathrm{OUTUBRO}+\mathrm{DE}+2002 . \mathrm{pdf} ? \mathrm{MOD}=\mathrm{AJPERES}$.

Acesso em 16.10.2014.

Dos Santos, L.B.; de Jesus Júnior, A.F.; Lima, J.C.; Fica Piras, P.R. (2020a). Formação técnica da juventude rural como elemento constituinte no processo de Indicação Geográfica do requeijão de Santa Bárbara. 11aㅡ FEBAFES. Disponível em https://feiradaagriculturafamiliar.com.br/wp-content/uploads/2020/12/EIXO-3Pablo-Rodrigo-Fica-Piras-Formacao-tecnica-da-juventude-rural-como-elementoconstituinte-no-processo-de-ig.pdf. Acesso em 08 de maio de 2021.

Dos Santos, L.B.; Menezes, R.R.L.; Leite, G.M.; Santos, Y.A.S.; Pereira, B.M.N.; ...; De Cerqueira, T.C.. (2020b). Onde moro, onde quero viver: Um olhar econômico, social e ambiental em tempos de pandemia. Série Requeijão Filosófico: on-line https://www.facebook.com/109277623747514/videos/702513877036526/.

Veiculado 22 de outubro de 2020.

Ducros, H. (2018). "Fête de la Soupe": rural identity, self-representation, and the (re)making of the village in France. Journal of Place Management and Development, 11(3):296-314.

FAO (2019). Geographical Indications for sustainable food systems, preserving and promoting agricultural and food heritage. Disponível em http://www.fao.org/3/ca5693en/ca5693en.pdf. Acesso em 11.11.2020.

GACC/UEFS - Gerência de Apoio aos Contratos e Convênios da Universidade Estadual de Feira de Santana. (2019) Termo de Convênio: "convênio que entre si celebram a Universidade Estadual de Feira de Santana e o município de Santa Bárbara".

IBGE (s.d). Portal das cidades e estados. Disponível em https://www.ibge.gov.br/cidades-e-estados/ba/santa-barbara.html. Acesso em 07 de fevereiro de 2020.

Kennedy, K. (2005). GATT 1994. In: Macrory, P.F.J.; Appleton, A.E.; Plummer, M.G. The World Trade Organization: legal, economic and political analysis, Vol.1. New York: Springer, ch. 5, $3135 \mathrm{p}$.

Legrand, W.; Sloan, P.; Fett, M.; Manten, T. (2019). The roles of terroir, food and gastronomy in destination authenticity. In: Dixit, S.K. (ed.) The Routledge Handbook of gastronomic tourism, ch.29. Routledge, New York, p.260-71.

Santos, M.S. e mais 34 autores. (2020) Santa Bárbara e os PET/MEC UEFS em sintonia com a Agenda 2030 da ONU. Pôster no XIX ENEPET 2019, São Luís, Maranhão.

SEBRAE. (2021). Oitenta novas indicações geográficas no país são identificadas com potencial de ser reconhecidas. Disponível em http://www.agenciasebrae.com.br/sites/asn/uf/NA/oitenta-novas-indicacoesgeograficas-no-pais-sao-identificadas-com-potencial-de-serreconhecidas,ce57811ca67a8710VgnVCM100000d701210aRCRD. Acesso em 09 de maio de 2021.

Sena, A.L.; Silva, J.S.; Oliveira, P.K.S.; Magalhães, P.P.G.; Silva, D.C.; Lima, J.R.O; Fica Piras, P.R.. (2018). Associação e qualificação de produtores como ingrediente 
do selo de origem no requeijão de Santa Bárbara. Pôster apresentado no XIII EBAPET, UEFS, Feira de Santana.

Souza, D.S.; Albinati, F.L.; Teshima, E.; Lobo, P.T.D.. (2019). Avaliação da qualidade microbiológica e físico-química do requeijão. Higiene Alimentar 33(288/289):23248.

Souza, M.J.A.; Souza, V.S.; Fica Piras, P.R.. (2017). Pertinencia de Chayanov y Gramsci para la permanencia campesina en la tierra: llamadas públicas y extensión universitaria. GT "05. Desarrollo Rural y cuestión agraria" del XXXI Congreso de la Asociación Latinoamericana de Sociología, Montevideo, 3 a 8 de diciembre de 2017.

Vandecandelaere, E.; Teyssier, C.; Barjolle, D.; Jeanneaux, P.; Fournier, S.; Beucherie, O. (2018). Strengthening sustainable food systems through geographical indications: an analysis of economic impacts. 13, European Bank for Reconstruction and Development (EBRD). 159 p. Disponível em https://hal.inrae.fr/hal-02788568/document. Acesso em 11.11.2020.

WTO - World Trade Organization. (n.d). Overview: the TRIPS Agreement. Disponível em https://www.wto.org/english/tratop e/trips e/intel2 e.htm\#: :text=The\%20TRIPS\%2 0Agreement\%20is\%20a,own\%20legal\%20system\%20and\%20practice. Acesso em 09 de maio de 2021.

\title{
GEOGRAPHICAL INDICATION PROCESS FOR REQUEIJÃO DE SANTA BÁRBARA AS A LINK BETWEEN THE TECHNICAL AND UNIVERSITY EDUCATION OF RURAL YOUTH.
}

\begin{abstract}
According to the Forum of Geographical Indications and Collective Brands, the Requeijão de Santa Bárbara is one of the fifteen products from Bahia that go in the direction of the registration request, along with stimulating innovation, competitiveness and local socioeconomic and technological development. Convergent with this, since May 21, 2018, in the inaugural class of the Technical Course in Food at the Colégio Estadual Professor Carlos Valadares, local educational initiatives identified professional training as a central part of the development of strategies to add value to local agricultural production and its derivatives. The bovine milk from Jacuípe Basin has as its most renowned destination the production of Requeijão in the city with which it is traditionally associated. Thus, the municipal institutional strategy for technical and professional qualification of local rural youth have been tied to the proximity to the University and its Engineering courses, as well as focusing on its convergence with the affirmation and consolidation of the Geographical Indication process of its Requeijão. This complex process contributes to add value and differentiate the product, to increase the technological standard and the job offer, to diversify the offer of services in sectors such as tourism, gastronomy and territorial marketing. There are antecedents of both the industrial and the local educational scenario, with information that intends to indicate the potential of a trajectory that fulfills the qualification requirements to fit the requirements that the legislation imposes.
\end{abstract}

Keywords: RDC275; Terroir; Technical and professional qualification; Value adding process; Local development. 